\title{
Functional characterization of developing heart in embryos using Electric Potential Sensors
}

\author{
E. Rendon-Morales, R. J. Prance, H. Prance \\ Sensor Technology Research Centre \\ School of Engineering and Informatics \\ University of Sussex \\ Brighton, UK \\ $\underline{\text { er241@sussex.ac.uk }}$
}

\author{
R. Aviles-Espinosa \\ Institute of Photonic Science \\ Mediterranean Technology Park, Av. Canal Olimpic s/n, \\ 08860 Castelldefels (Barcelona), Spain
}

\begin{abstract}
The characterization of the electrocardiographic activity of the living zebrafish heart during early developmental stages is a challenging task. Most of the available techniques are limited to heartbeat rate quantification being this inaccurate. Other invasive methodologies require the insertion of electrodes noise isolated environments and advanced amplification stages making these techniques very expensive. In this paper, we present a novel and non-invasive sensor development to characterize the functional activity of the developing heart of in vivo zebrafish embryos. The design is based on the Electric Potential Sensing technology patented at Sussex which has been developed to achieve reproducibility and continuous detection. We present preliminary functional characterization data of the developing zebrafish heart starting at 3 days-post-fertilization. Results show that using the proposed system for mapping the electrocardiographic activity of the zebrafish heart at early developmental stages is successfully accomplished. This is the first time that such a sensitive sensor has been developed for measuring the electrical changes occurring on micron sized $(<$ $100 \mu \mathrm{m})$ living samples such as the zebrafish heart
\end{abstract}

Keywords - Biomedical sensors, cardiology, embryionic heart, Electric Potential Sensors, electrophysiology and electrocardiogram.

\section{INTRODUCTION}

The study of the electrical properties of the heart at early developmental stages is of scientific relevance, as this potentially could allow gaining insight on the originating causes of heart repolarization abnormalities such as congenital cardiac arrhythmias and long/short QT syndrome, costing actually the EU economy almost 196 million a year [1].

In the last years, the zebrafish (Danio rerio) has been considered by researchers as a powerful model organism for the study of heart related diseases. This is mainly because zebrafish electrocardiogram (ECG) waveform possesses a remarkable resemblance to those of humans [2]. Although its heart morphology has only a two-chambered heart (compared with the four-chambered human heart) and a simple fully functioning circulatory system, the zebrafish has been suggested as a useful model organism for studies of human heart development and cardiac patho-electrophysiology. A major advantage of the zebrafish is that it has an already beating heart after just 24 hours post-fertilization, thus enabling assessment of its cardiac activity relatively early. Another advantage of the zebrafish for heart abnormality studies is it that the embryo is transparent, allowing for easy assessment of heart development, heart rate analysis and phenotypic characterization

Recording electrophysiological activity from embryonic heart is a challenging task as it requires the design of highly accurate instrumentation tools that produce no perturbation of the parameter of interest. This is essential for enhancing the ability to clearly distinguish cardiac events observed in ECG traces. Given that the amplitude of the targeted signal is within the orders of $\mu \mathrm{V}$, a controlled environment is required that considers also the challenges posed by the saline buffer in which the embryo is kept alive. In addition the electrode design should allow precise positioning, as this is a crucial step for obtaining reproducible signals [4].

The most common methodologies for characterizing zebrafish cardiac activity are limited to quantifying the cardiac rate (i.e. including simple visual inspections). This has the disadvantage of being time consuming and operator dependent. On the other hand, methodologies that require heart explantation techniques have been used for recording the electrophysiological activity from zebrafish heart. However these are invasive and have been performed on adult samples [3, 5]. Alternatively, more advanced techniques based on imaging procedures have been implemented through complex image recognition algorithms that assess the time-varying presence of blood cells in the heart chamber during the cardiac cycle [6]. Particularly, this technique was developed for assessing zebrafish embryos and pre-feeding stage larvae, however, it is limited to quantify the cardiac rate. Finally, the use of other techniques including those incorporating advanced amplifiers and digital signal processing still require noise isolated environments [7] making them expensive to use.

In this paper we report a novel device that enables performing functional characterization of the developing heart in zebrafish embryos using Electric Potential Sensors (EPS). The instrumentation design has been developed for signal recording reproducibility, enable continuous detection and to be truly non-invasive. This proof of concept demonstration shows how using our non-invasive approach based on electric field detection it is possible to obtain electrocardiographic measurements from embryonic zebrafish heart. Zebrafish embryos (referred here as $0-5 \mathrm{dpf}$ ) are not regulated under the 
UK Specific procedure Act 1986 and European Directive 2010/63/EU and thus carry reduced bioethical limitations.

We present preliminary results using the new sensor development based on the EPS technology, which enables the acquisition of electrophysiological signals from in vivo zebrafish heart embryos starting at 3 days-post-fertilization (dpf). Remarkable measurements were recorded without requiring any averaging or post-processing techniques and more importantly maintaining the embryo alive. Results show that the proposed system is capable of mapping electrocardiographic activity from the zebrafish heart at early developmental stages. This is the first time to the best of our knowledge that such a sensitive sensor has been developed for measuring the electrical changes occurring on micron sized (< $100 \mu \mathrm{m}$ ) living samples such as the zebrafish heart.

This paper is organized as follows; in Section II a brief overview of EPS sensing technology is presented. Section III describes the main electronic design considerations to assess electrocardiographic activity in zebrafish embryos. In Section IV examples of the recorded ECGs are presented. Finally, conclusions are drawn in Section V.

\section{ELECTRIC POTENTIAL SENSORS}

The EPS sensor was invented and patented at the University of Sussex as a non-invasive sensing technology [8]. Its electric potential sensing capability has been demonstrated in several applications such as body electrophysiology: characterizing human electro-cardiogram (ECG) in situ [9] and remotely [10], electroencephalogram (EEG) [11] human hydration assessment [12], non-destructive testing of composite materials [13], imaging electrical activity of integrated circuits [14], following the propagation of pulses in saline solutions [15], novel nuclear magnetic resonance (NMR or MRI) sensing probes real-time array imaging [16], and imaging electrostatic fingerprints [17].

The EPS sensor is a feedback enhanced and stabilized electrometer-based amplifier that operates based on displacement current measurements. This means that no real charge current is applied, eliminating the need to make direct electrical contact with the sample surface. This result in a completely bio-compatible novel non-invasive instrumentation tool. The displacement current concept was first postulated by J.C. Maxwell in 1861 to maintain conservation of charge produced by electric fields in an electric capacitor circuit. The EPS sensor falls in the capacitive sensor category, used to noninvasively sense the displacement currents induced by timevarying electric fields. The development of the electronics required for these sensors has been discussed in detail in previous publications $[17,18]$.

The EPS sensor design encompasses an external bias circuitry in a way that does not compromise the input impedance of the sensor. It includes associated feedback loops providing the functions of bootstrapping, neutralization and guarding to enhance the input impedance, reduce the input capacitance and maintain the electronic stability of the sensor [18]. The net effect of this combination of positive feedback techniques is to produce a broadband sensor within the range up to $100 \mathrm{MHz}$, with extremely high input impedance (up to $\left.10^{18} \Omega\right)$ and low effective input capacitance $\left(\sim 10^{-15} \mathrm{~F}\right)$. This characteristic is crucial in achieving a small coupling capacitance with the sample. In essence, we have applied novel feedback techniques to a low noise electrometer amplifier aiming to satisfy its bias requirements while at the same time increasing the input impedance of the sensor front-end. This provides both: a minimum loading of the source by the sensor amplifier and allows the rejection of unwanted interference signals. This combination results in a highly sensitive sensor at the operating frequencies of most electrophysiological signals. Its performance as a non-perturbative detector has also been demonstrated in [19] being able to measure fields as low as 2.6 $\mu \mathrm{V} / \mathrm{m}$ with $2 \%$ accuracy.

The actual EPS operating bandwidth is dependent on the application and determined both by the choice of amplifier and by the coupling capacitance between the sensor and the source. The EPS is capable of characterizing changes in spatial potential or electric field considering only displacement currents flowing within a source. Due to its weak capacitive coupling, it is possible to measure even though an insulating surface layer.

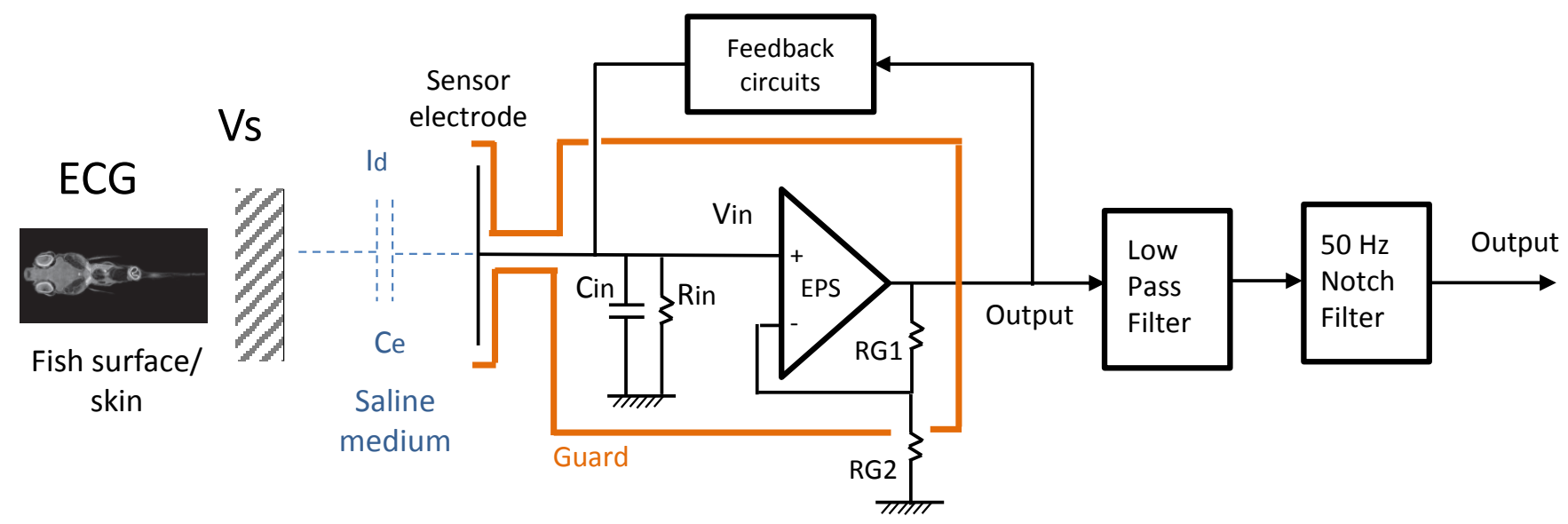

Fig 1. EPS sensor design showing the relevant guarding technique from the electrode interface and the amplification process [25] 


\section{EPS SENSOR DESIGN FOR ZEBRAFISH EMBRYOS}

The EPS sensor presented here is designed to characterize the electrocardiographic activity of developing heart in zebrafish embryos. It uses an ultra-high input impedance to increase the sensitivity of the electrode while reducing electric potentials at the input of the sensor amplifier. Fig. 1 shows the EPS sensor design including the relevant guarding technique from the electrode interface and the amplification and filtering processes [25]. It was built using a modified ultra-high input impedance EPS sensor, which has been tuned for rejecting external noise at particular frequencies $(<50 \mathrm{~Hz})$. For this design we have particularly used a hardware Notch filter and a Comb filter (not shown).

To perform the characterization of electrophysiological signals from living zebrafish, it is important to consider additional challenges which are not present when recording human surface ECG. For example, the electrical conductivity of the saline buffer where the sample is kept alive, makes challenging to retrieve the electrical information directly from the sample.

In a previous work [15], we carried out several experiments for identifying the effects of the electrical conductivity of the saline buffer as an interfacing medium when using the EPS sensor. We assembled an experimental cell to measure the propagation delay of electrical signals though a small coupling capacitance in a saline buffer. The results obtained from such work confirm the capability of the EPS sensor to detect signals up to $70 \mathrm{mV}$ while immersed in a saline buffer. This proof of concept demonstration became the first step towards developing a non-invasive sensor for measuring physiological parameters in biological organisms immersed in a saline buffer.

The most important characteristic of the EPS sensor design it is that the combination of such a high input impedance, active front-end with an insulating electrode means that the EPS sensor draws no real current from the source and can be categorized as such an 'ideal voltmeter' which makes it a noninvasive tool for measuring the electric field variations inside zebrafish embryos.
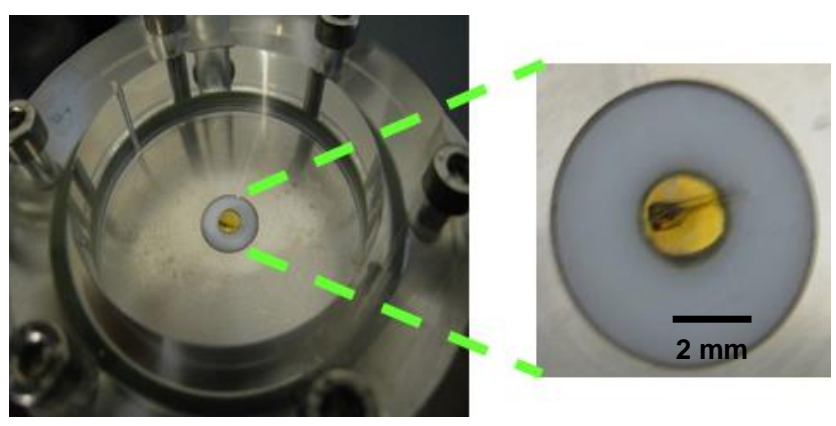

Fig 2. Picture of the mini fish tank based on the Sussex patented EPS electrode. It contains the sensing electrode on the center including the amplification circuitry

\section{A. Experimental set up}

A picture of the novel sensor design for recording in vivo zebrafish electrocardiographic activity non-invasively is shown in Fig 2. The design incorporates a mini fish tank which includes an integrated electro active EPS sensor with internal input bias current circuitry and guarding. Fig 2 also shows the representation of the mini fish tank containing the central EPS sensor using a $2 \mathrm{~mm}$ diameter sensing electrode. The second reference electrode is connected through the shield of the central electrode making contact with the surrounding medium during our recordings. The EPS sensor has been developed in combination with commercially available electrometer amplifiers incorporating electronic feedback techniques in order to increase the input impedance and maintain stability.

The voltage outputs from the sensors are fed to an analogue processing stage where the signals are amplified and band-pass filtered $(1 \mathrm{~Hz}$ to $100 \mathrm{~Hz})$. This modified version of the sensor has been used for characterizing zebrafish ECG signals reported in this paper (See Fig 1). The output of the processor is fed to a commercial NI data acquisition system having a maximum sampling rate of $10 \mathrm{kS} / \mathrm{s}$ [22]. The data is then acquired on a laptop computer. Display and storage of the digital data is controlled using a custom made graphical user interface based on LabVIEW software [23]. Using the proposed prototype a remarkable noise level of $70 \mathrm{nV} \mathrm{Hz}^{-1 / 2}$ at the $1 \mathrm{~Hz}$ measuring bandwidth (referred to the input) reducing to $30 \mathrm{nV} \mathrm{Hz}$-1/2 at the $100 \mathrm{~Hz}$ measuring bandwidth. The system low noise performance is of great importance when sensing small ECG amplitudes from living zebrafish heart.

Finally, the proposed setup incorporates a flexible design that could be adapted to any existing microscope system, including a user-application interface to record ECG signals. In order to control any kind of moving artefacts from zebrafish samples during the ECG characterization, we visualize embryos using a custom made bright field microscope, based on an Olympus 4X NA 0.1 objective equipped with a monochrome camera placed on top of the experimental set-up.

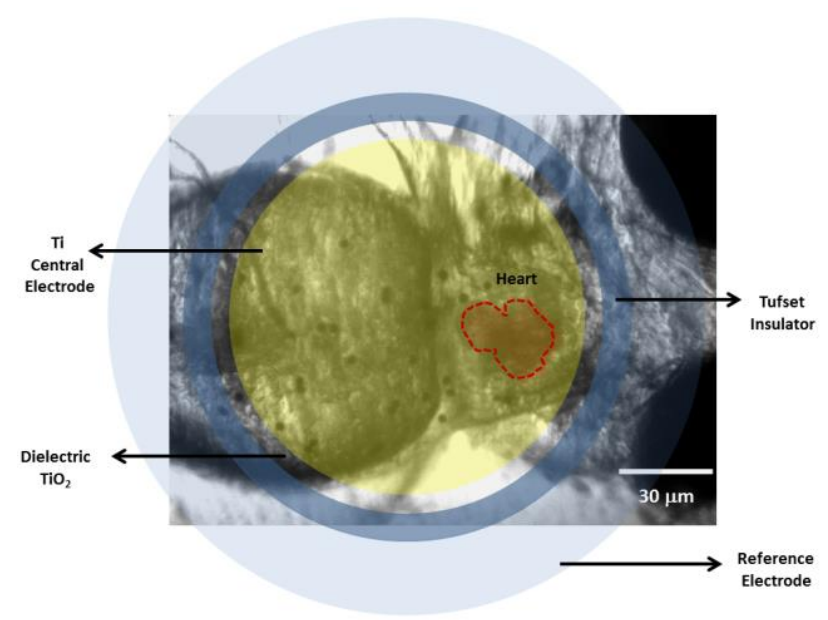

Fig 3. Top view representation of the Ti based central EPS electrode for detecting electrocardiogram activity of in vivo zebrafish embryos. It shows an example of the zebrafish heart image acquired using an Olympus $4 \mathrm{X}$ objective included as a part of the exprimental setup. Scale bar applies for the Zebrafish dimensions. 


\section{B. Electrode interface}

A crucial consideration during the design of the sensor front-end, it is the electrode material interface. Currently, there are several types of electrodes used for recording electrophysiological measurements. The most common interface that has been extensively used during the last decade it based on silver/silver chloride ( $\mathrm{Ag}-\mathrm{AgCl})$.

The main drawback of using $\mathrm{Ag}-\mathrm{AgCl}$ electrodes it is invasive nature as they require to have electrical contact with the sample. Due to this, the current that flows through them should be carefully controlled aiming to avoid exhausting the $\mathrm{AgCl}$ material as this could produce toxic effects on many proteins inside the sample. An additional disadvantage of the $\mathrm{Ag}-\mathrm{AgCl}$ electrodes is that there are poorly reversible causing electrode polarization.

It is worth mentioning that contrary to $\mathrm{Ag}-\mathrm{AgCl}$ electrodes, the EPS requires only making physical contact with the sample. Therefore it is not affected by variations due to contact resistance in the same way that occurs within traditional electrodes.

The electrode interface presented here was developed using a metallic titanium (Ti) based central electrode coated with a titanium dioxide $\left(\mathrm{TiO}_{2}\right)$ substrate acting as a dielectric. This combination as part of the EPS hardware circuitry provides a sensor with excellent biocompatibility properties [21]. This makes possible the detection of electric field measurements generated by the in vivo zebrafish heart immersed in E3 saline buffer (Sigma Aldrich). Fig. 3 shows the top view representation of the $\mathrm{Ti}$ based central EPS electrode for detecting electrocardiographic activity of in vivo zebrafish embryos. In addition to this an image acquired using our custom built microscope using $4 \mathrm{x}$ magnification is shown. Please note that the heart has been outlined for simplifying its identification.

\section{RESULS}

In this paper, we present relevant results to demonstrate that using our new sensor development, we are able to perform the functional characterization of developing heart in embryos immersed inside a saline buffer environment [25]. We carried out several experiments, considering embryonic zebrafish samples (3 and $5 \mathrm{dpf}$ ). Wild type Zebrafish embryos were raised and kept under standard laboratory conditions at 28.5 C. Embryos were collected during the morning and then transferred immediately to E3 embryo medium (Sigma Aldrich [20]), which was changed daily.

Experiments were performed at room temperature $\left(22^{\circ} \mathrm{C}\right)$. Embryos were initially acclimatized before the recordings took place. During ECG recordings, embryonic zebrafish were also anesthetized using Tricaine methanesulfonate $(0.3 \mathrm{mg} / \mathrm{ml}$ Sigma) $5 \mathrm{~min}$ before these were transferred into $3 \mathrm{ml}$ of E3 embryo medium inside the mini fish tank. The embryo was positioned ventrally touching the surface of the central electrode. Recordings were taken for 1 minute. In some cases it was necessary to apply a slight pressure over the surface of the fish using a tip of glass capillary tube, to enhance contact with the central electrode. Once the recordings finished, the embryo was transferred to its original E3 embryo medium, in which after several minutes once the anesthetic effect has passed, we confirmed that the sample was still alive.

For the proposed experiments, the setup was located in a laboratory environment, surrounded by $50 \mathrm{~Hz}$ line operated equipment. The sensitivity of the presented version of the EPS sensor is such that under normal circumstances the device would saturate with $50 \mathrm{~Hz}$ and related harmonics preventing the acquisition of signals. However, due to the active filtering stages and feedback techniques applied within its design, the EPS is capable of rejecting the principal components of line related noise, with $50 \mathrm{~dB}$ of rejection at $50 \mathrm{~Hz}$.

Fig 4 shows an example of the raw data collected from a 5 dpf zebrafish embryo during 10 seconds. Please note that gain values from the amplification stages have been considered aiming to characterize the real amplitude of the obtained signals. We have repeated these experiments using more samples being able to confirm the presented results. The recorded mean heart rate was $156 \mathrm{bpm}$ having a mean peak amplitude average of $28.3 \pm 3 \mu \mathrm{V}$.

\section{ECG signal of an in vivo 5 dpf zebrafish}

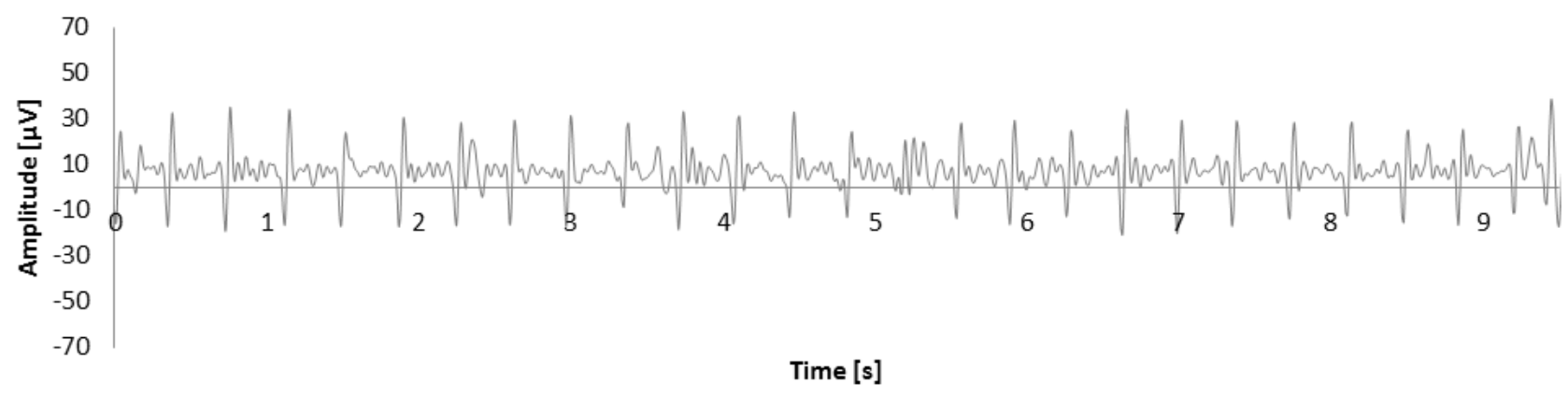

Fig 4. Data collected during 10 seconds from a 5 dpf zebrafish embryo using the EPS sensor. 


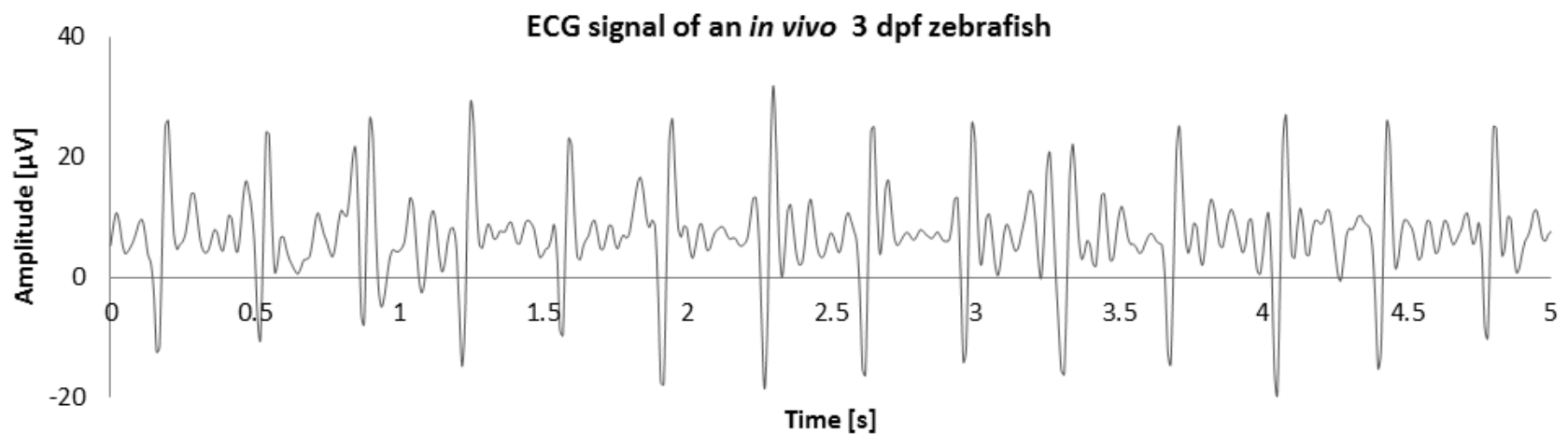

Fig 5. Data collected using the EPS sensor from a 3 dpf zebrafish embryo.

By comparison Fig 5 shows an example of a cardiac signal obtained from a $3 \mathrm{dpf}$ zebrafish embryo. The mean heart rate of this embryo is $169 \mathrm{bpm}$ having a mean peak amplitude average of $22.5 \pm 2 \mu \mathrm{V}$. The acquired signals are collected from the EPS sensor with an operating bandwidth of $1 \mathrm{~Hz}$ to $100 \mathrm{~Hz}$. The data was recorded in real-time with no signal averaging or post-processing techniques applied. As it is shown in Fig 4 and Fig 5, the similarity between these results is indicative of the main electrical nature of the signal [25].

The results presented in Fig. 4 and 5 clearly confirm the time-based relationship between the data collected using EPS sensor and other works using conventional $\mathrm{Ag}-\mathrm{AgCl}$ electrodes, suggesting that surface ECG recordings using the EPS sensor are a true representation of the electrical activity of the heart. In addition the main peak component in the recorded ECG data is coincident with the arterial pulse and frequency determined using imaging algorithms reported on [6].

Using the novel non-invasive EPS sensor, we have been able to satisfactory characterize the electric fields present in zebrafish embryos heart starting from $3 \mathrm{dpf}$. We have carried out this by measuring the potential generated across zebrafish samples presenting several examples of heart beat rate values together with the raw data recorded in real-time. This demonstrates the capability of our sensor to characterize passively ECG signals with the here presented technique.

\section{CONCLUSIONS}

In summary, we have presented a new sensor development based on the EPS technology to characterize ECG activity of developing hearts in embryos non-invasively. Results confirm that using our device it is possible to achieve high quality ECG recordings from zebrafish heart during early developmental stages starting at $3 \mathrm{dpf}$. Highly sensitive detection of ECG signals was accomplished using the proposed EPS sensor even in the presence of $50 \mathrm{~Hz}$ without no post-processing or signal averaging techniques applied. To the best of our knowledge, this is the first time that such characterization have been demonstrated for in vivo zebrafish embryos non-invasively using our technique based on displacement current measurements.
Future directions of this research are focused on extending these laboratory experiments, aiming to develop a multiplatform based on EPS arrays for the simultaneous analysis of ECG variations (i.e. heart rate variability), which can be used for studying the cardio-toxic and neuro-toxic effects of pharmacological compounds in drug discovery.

\section{ACKNOWLEDGMENTS}

Authors would like to thank Martin Nock from STRC for his valuable discussion and support. Special thanks to Professor Leon Lagnado and Sofie-Helene Seibel from Sussex Neuroscience, for providing zebrafish samples. This work was partially funded by Marie Skłodowska-Curie actions COFUND with the Department of Economy and Knowledge from the Generalitat de Catalunya.

\section{REFERENCES}

[1] N Townsend et al, Coronary heart disease statistics (British Heart Foundation, London 2012).

[2] A. O. Verkerk and C. A. Remme, Frontiers in physiology 3, 255 (2012).

[3] D. J. Milan, I. L. Jones, P. T. Ellinor, and C. A. MacRae, American journal of physiology. Heart and circulatory physiology 291 (1), H269 (2006)

[4] Jaakko Malmivuo \& Robert Plonsey, Bioelectromagnetism - Principles and Applications of Bioelectric and Biomagnetic Fields (Oxford University Press, New York 1995) p.47.

[5] C. T. Tsai, C. K. Wu, F. T. Chiang, C. D. Tseng, J. K. Lee, C. C. Yu, Y. C. Wang, L. P. Lai, J. L. Lin, and J. J. Hwang, Clinica chimica acta; international journal of clinical chemistry 412 (21-22), 1963 (2011).

[6] Elisa De Luca, Gian Maria Zaccaria, Marwa Hadhoud, Giovanna Rizzo, Raffaele Ponzini, Umberto Morbiducci, and Massimo Mattia Santoro, Scientific Reports (2014).

[7] S. S. Dhillon, E. Doro, I. Magyary, S. Egginton, A. Sik, and F. Muller, PloS one 8 (4), e60552 (2013).

[8] R. Prance et al., U.S. patent 20090167324 3,508,029 (2 July 2009).

[9] C. J. Harland, T. D. Clark, N. S. Peters, M. J. Everitt, and P. B. Stiffell, Physiological measurement 26 (6), 939 (2005).

[10] R. J. Prance, S. T. Beardsmore-Rust, P. Watson, C. J. Harland, and H. Prance, Applied Physics Letters 93 (3), 033906 (2008).

[11] M. Fatoorechi, J. Parkinson, R. J. Prance, H. Prance, A. K. Seth, and D. J. Schwartzman, Journal of neuroscience methods 251, 7 (2015).

[12] E. Rendon-Morales, et al. "Towards the correlation of human hydration with the electrical activity of the heart using EPS sensor" in proceedings of the IEEE, Zadar Croatia Sensor Applications Sym. 13-15 April 2015. 
[13] A. Aydin, R. J. Prance, H. Prance, and C. J. Harland, Applied Physics Letters 95 (12), 124102 (2009).

[14] W. Gebrial, R. J. Prance, C. J. Harland, and T. D. Clark, Review of Scientific Instruments 77 (6), 063708 (2006)

[15] W. Gebrial, R. J. Prance, C. J. Harland, C. Antrobus, and T. D. Clark, Journal of Physics D: Applied Physics 40 (1), 31 (2007).

[16] R. J. Prance and A. Aydin, Appl. Phys. Lett. 91, $044103 \_2007$

[17] P. Watson, R. J. Prance, S. T. Beardsmore-Rust, and H. Prance, Forensic science international 209 (1-3), e41 (2011).

[18] Helen Prance, Sensor Developments for Electrophysiological Characterizeing in Healthcare. (2011).

[19] A. Aydin, P. B. Stiffell, R. J. Prance, and H. Prance, Measurement Science and Technology 21 (12), 125901 (2010).
[20] Buffer Solutions 2015 Sigma-Aldrich Co. LLC.

[21] Z. F. Yin, L. Wu, H. G. Yang, and Y. H. Su, Physical chemistry chemical physics : PCCP 15 (14), 4844 (2013).

[22] NI data acquisition system DAQ NI USB 6008 card.

[23] LabVIEW graphical programming language (National Instruments, Newbury Berkshire, RG14 5SJ, UK.1)

[24] Netter (1971): The generation of the ECG signal in the Einthoven limb leads

[25] Rendon-Morales, Elizabeth, Prance, Robert, Prance, Helen and AvilesEspinosa, Rodrigo (2015) Non-invasive electrocardiogram detection of in vivo zebrafish embryos using electric potential sensors. Applied Physics Letters, 107 (19). 